\title{
Parton Distributions from HERA
}

\author{
Voica Radescu ${ }^{1}$ \\ Physikalisches Institut, University of Heidelberg, \\ E-mail:voica@mail.desy.de
}

\begin{abstract}
A QCD fit analysis to the combined HERA-I inclusive deep inelastic cross sections measured by the $\mathrm{H} 1$ and ZEUS collaborations for $e^{ \pm} p$ scattering resulting into a competitive NLO PDF set, HERAPDF1.0 is presented. HERAPDF at NNLO fits are presented as well, resulting, however, in a worse description of the combined HERA data. In addition, the QCD fit analysis of the combined HERA-I inclusive deep inelastic cross sections has been extended to include combined HERA II measurements at high $Q^{2}$. The effect of including these data on the determination of parton distribution functions is analysed, resulting into HERAPDF1.5. The precision of the new PDFs at high-X is considerably improved, particularly in the valence sector. The predictions based on HERAPDF1.0 and HERAPDF1.5 are compared to $p \bar{p}$ processes at Tevatron, and also used for predictions of the $p p$ processes at the LHC.
\end{abstract}

Keywords: QCD, PDFs, proton structure, DIS, HERA, fits

PACS: $12.38 . \mathrm{Qk}, 12.38 .-\mathrm{t}, 14.65 .-\mathrm{q}$

\section{COMBINED H1 AND ZEUS CROSS SECTION MEASUREMENTS}

Deep inelastic scattering (DIS) at HERA has been crucial to the exploration of proton structure and quark-gluon interaction dynamics. The combination of the H1 and ZEUS data provides the most accurate measurements of DIS inclusive double differential crosssections of neutral (NC) and charged current (CC) $e^{ \pm} p$ scattering over an extended kinematic range of $0.045<Q^{2}<30000 \mathrm{GeV}^{2}$ and $6 \times 10^{-5}<x<0.65$. Therefore, these accurate measurements can be used as a sole input to the QCD analysis to determine the proton structure - parton distribution functions (PDFs) as described in the following sections, which can be used then for precise predictions for the LHC processes.

The combination of data uses the $\chi^{2}$ minimisation method and it takes into account the correlated systematic uncertainties for the $\mathrm{H} 1$ and ZEUS cross-section measurements [1]. This combination procedure is applied to the published inclusive deep inelastic cross sections measured by the $\mathrm{H} 1$ and ZEUS collaborations in CC and NC unpolarised $e p$ scattering at HERA during the period 1994-2000, termed HERA I; and to the published ZEUS data for $\mathrm{CC}$ and $\mathrm{NC} e \pm p$ scattering from entire running period together with the preliminary data of high $Q 2$ measured by the H1 during the entire running period of HERA, which considerably improve PDF uncertainties at high- $x$ as described in these proceedings.

\footnotetext{
${ }^{1}$ On behalf of the $\mathrm{H} 1$ and ZEUS collaborations
} 


\section{QCD ANALYSIS SETTINGS}

The above combined data is used as a sole input into a QCD fit analysis to extract the proton's PDFs. The HERA data have a minimum invariant mass of the hadronic system, $W$, of $15 \mathrm{GeV}$ and a maximum $x$ of 0.65 , such that they are in a kinematic region where there is no sensitivity to target mass and large- $x$ higher-twist contributions. In addition, to restrain to the region where perturbative $\mathrm{QCD}$ is valid, only data above $Q_{\min }^{2}=3.5 \mathrm{GeV}^{2}$ is used in the central fit.

The fit procedure consists first in parametrising PDFs at a starting scale $Q_{0}^{2}=$ $1.9 \mathrm{GeV}^{2}$, chosen to be below the charm mass threshold. The parametrised PDFs are the valence distributions $x u_{v}$ and $x d_{v}$, the gluon distribution $x g$, and the $u$-type and $d$ type $x \bar{U}, x \bar{D}$, where $x \bar{U}=x \bar{u}, x \bar{D}=x \bar{d}+x \bar{s}$. The following standard functional form is used to parametrise them

$$
x f(x)=A x^{B}(1-x)^{C}\left(1+D x+E x^{2}\right),
$$

where the normalisation parameters, $A_{u v}, A_{d v}, A_{g}$, are constrained by the QCD sum-rules. The $B$ parameters $B_{\bar{U}}$ and $B_{\bar{D}}$ are set equal, $B_{\bar{U}}=B_{\bar{D}}$, such that there is a single $B$ parameter for the sea distributions. The strange quark distribution is already present at the starting scale and it is assumed here that $x \bar{s}=f_{s} x \bar{D}$ at $Q_{0}^{2}$. The strange fraction is chosen to be $f_{s}=0.31$ which is consistent with determinations of this fraction using neutrino induced di-muon production. In addition, to ensure that $x \bar{u} \rightarrow x \bar{d}$ as $x \rightarrow 0$, $A_{\bar{U}}=A_{\bar{D}}\left(1-f_{s}\right)$. The $D$ and $E$ are introduced one by one until further improvement in $\chi^{2}$ is found. The best fit results in a total of 10 free parameters.

The PDFs are then evolved using DGLAP evolution equations [5]. at NLO and NNLO in the $\overline{M S}$ scheme with the renormalisation and factorisation scales set to $Q^{2}$. The QCD predictions for the structure functions are obtained by convoluting the PDFs with the calculable coefficient functions taking into account mass effect for the heavy quarks based on the general mass variable flavour scheme [4].

The uncertainties at HERA are classified in three categories: experimental, model, and parametrisation uncertainties. The consistency of the input data set and its small systematic uncertainties enable us to calculate the experimental uncertainties on the PDFs using the $\chi^{2}$ tolerance $\Delta \chi^{2}=1$. The model uncertainties are evaluated by varying the input assumptions, which are the variation of the starting scale and of the $Q_{\min }^{2}$, the variations of the heavy quark masses which are set to the standard values of $m_{c}=1.4 \mathrm{GeV}$ and $m_{b}=4.75 \mathrm{GeV}$ for the central fit, and the variation of $f_{s}$. The parametrisation uncertainty is estimated as an envelope which is formed as a maximal deviation at each $x$ value from the central fit of 10 parameter fits with $D$ and $E$ non-zero from Equation 1.

\section{RESULTS AND COMPARISONS}

The NLO QCD analysis has been performed first to the final HERA I data resulting into HERAPDF1.0 which has been published [2] and it will be used as a reference for the new studies. The NNLO fit results have been also performed using the same scheme as used for MSTW PDF sets, for different values of the strong coupling, $\alpha_{s}\left(M_{Z}\right)=0.1176$ 
and $\alpha_{S}\left(M_{Z}\right)=0.1145$ [6]. Fit results are shown in Figure 1. However, the NNLO fits, when perfomed under the same settings and parametrisation, do not bring improvement in terms of the fit quality with respect to NLO fits (worse by about 65 and 50 units of $\chi^{2}$, respectively) for both $\alpha_{S}$ cases, with a preference for lower value of the strong coupling at NNLO.
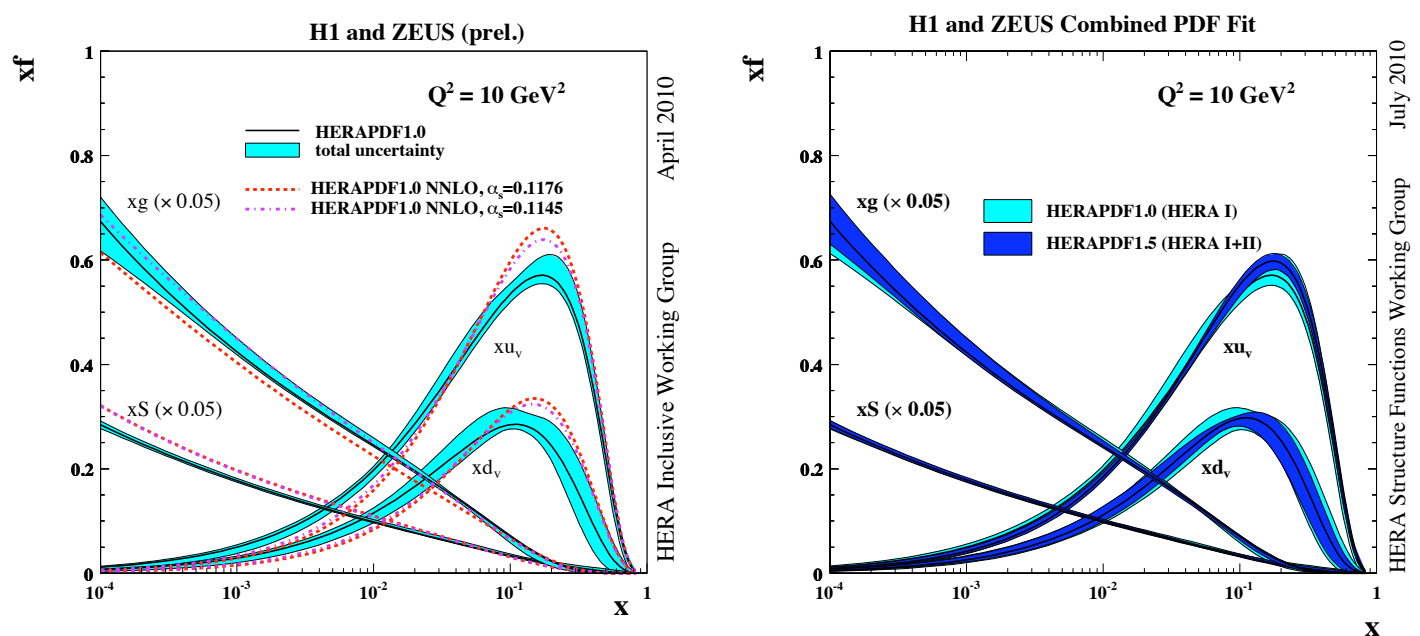

FIGURE 1. Figure shows on the left hand side the summary plot at the $Q^{2}=10 \mathrm{GeV}^{2}$ with gluon, sea (which are scaled by a factor of 0.05) and the valence distributions for HERAPDF1.0 at NLO with its three sources of uncertainties: experimental (inner band), model (lightest colored band), and parametrisation (outer band). On the right hand side, it is shown the comparison between the HERAPDF1.0 (light color) based on HERA I data and HERAPDF1.5 (dark color) based on HERA I and II data, using total uncertainty band at $Q^{2}=10 \mathrm{GeV}^{2}$

The QCD fit analysis of the combined HERA-I inclusive deep inelastic cross sections has been extended to include combined HERA II measurements at high $Q^{2}$ resulting into HERAPDF1.5 [6]. Figure 3 shows that the precision of the PDFs at high- $x$ is considerably improved, not only for the experimental uncertainties, but also for the parametrisation uncertainty - particularly in the valence sector, when compared to HERAPDF1.0 displayed in Figure 2.

Of particular interest for this workshop is also the $d / u$ ratio, therefore the impact of the new data on this distribution is shown as well in Figure 4, with the visibly improved uncertainties once the more precise HERA II data is included in the fit. The predictions based on HERAPDFs from the DIS process agree well with the Tevatron jet production, $Z$ and $W$ cross sections from the $p \bar{p}$ process as shown in Figure 5 and provide a competitive prediction for the LHC $p p$ processes as illustrated in Figure 6 and, after including the HERA II in the fit, in Figure 7. 

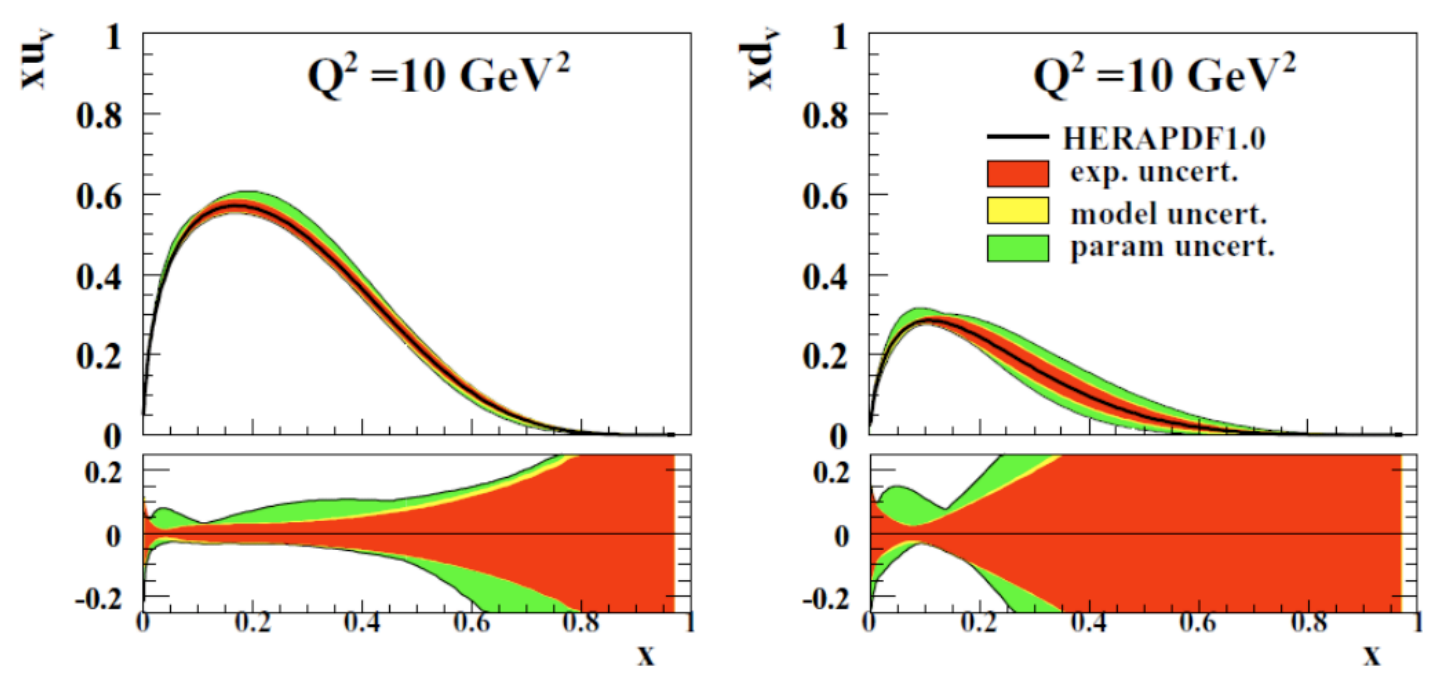

FIGURE 2. Figure shows the valence distributions and their relative uncertainties for the HERAPDF1.0 at the $Q^{2}=10 \mathrm{GeV}^{2}$. The errors include the experimental (red), model (yellow) and the PDF parametrisation (green) uncertainties.
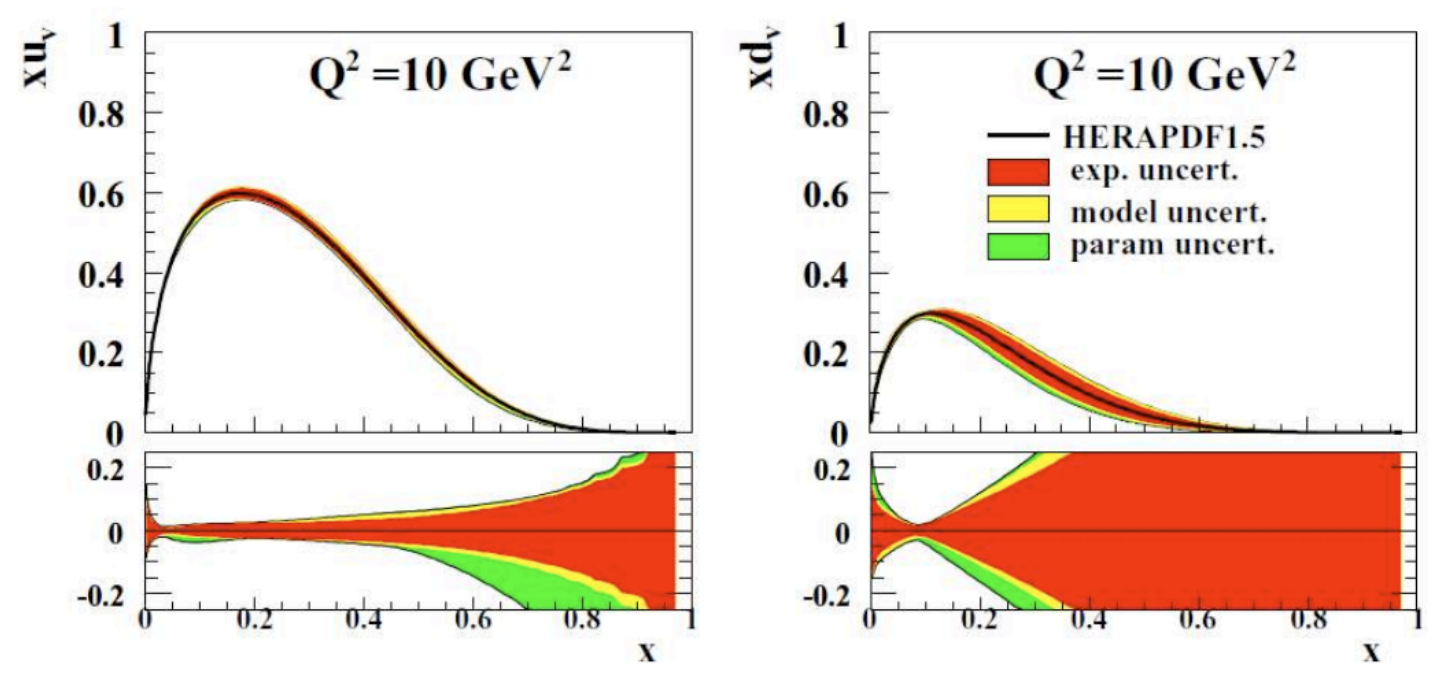

FIGURE 3. Figure shows the same valence distributions as in Figure 2 for the HERAPDF1.5, resulting in an improved precision compared to HERAPDF1.0.

\section{REFERENCES}

1. H1 Collaboration, F. Aaron et al. Eur.Phys.J.C64 (2009) 561, arXiv 0904.0929.

2. H1 Collaboration, ZEUS Collaboration, F. Aaron et al., JHEP 1001, 109 (2010), arXiv:0911.0884.

3. C. Amsler et al. (Particle Data Group), Phys. Lett. B667, (2008).

4. R. S. Thorne code, revised in 2008.

5. QCDNUM package, M. Botje, (2010), arXiv:1005.1481, http://www.nikef.nl/h24/qcdnum/index.html

6. The LHAPDF grid files are located at https://www.desy.de/h1 zeus/combined_results/index.php?do=proton_structure 

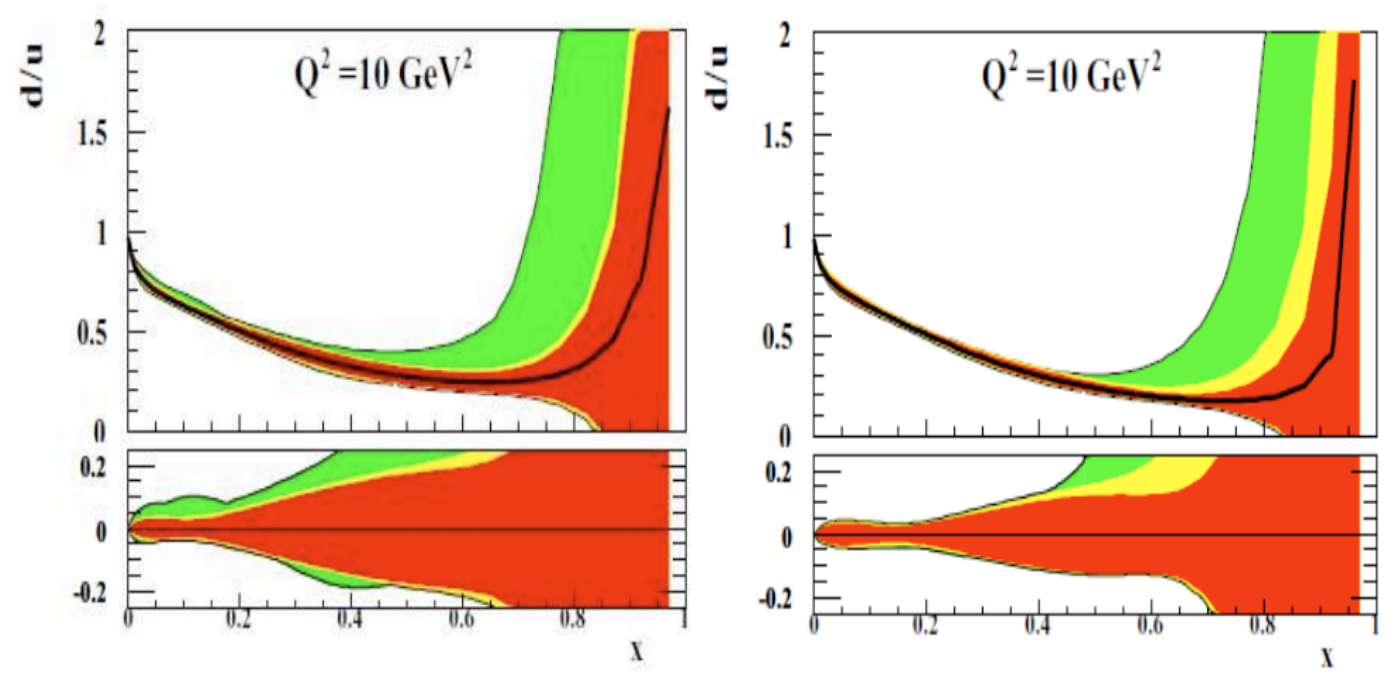

FIGURE 4. Figure shows on the left hand side the $d / u$ ratio distribution and its relative uncertainties for the HERAPDF1.0 at the $Q^{2}=10 \mathrm{GeV}^{2}$. The errors include the experimental (red), model (yellow) and the PDF parametrisation (green) uncertainties. On the right hand side the same distribution for the HERAPDF1.5, with an improved precision compared to HERAPDF1.0.
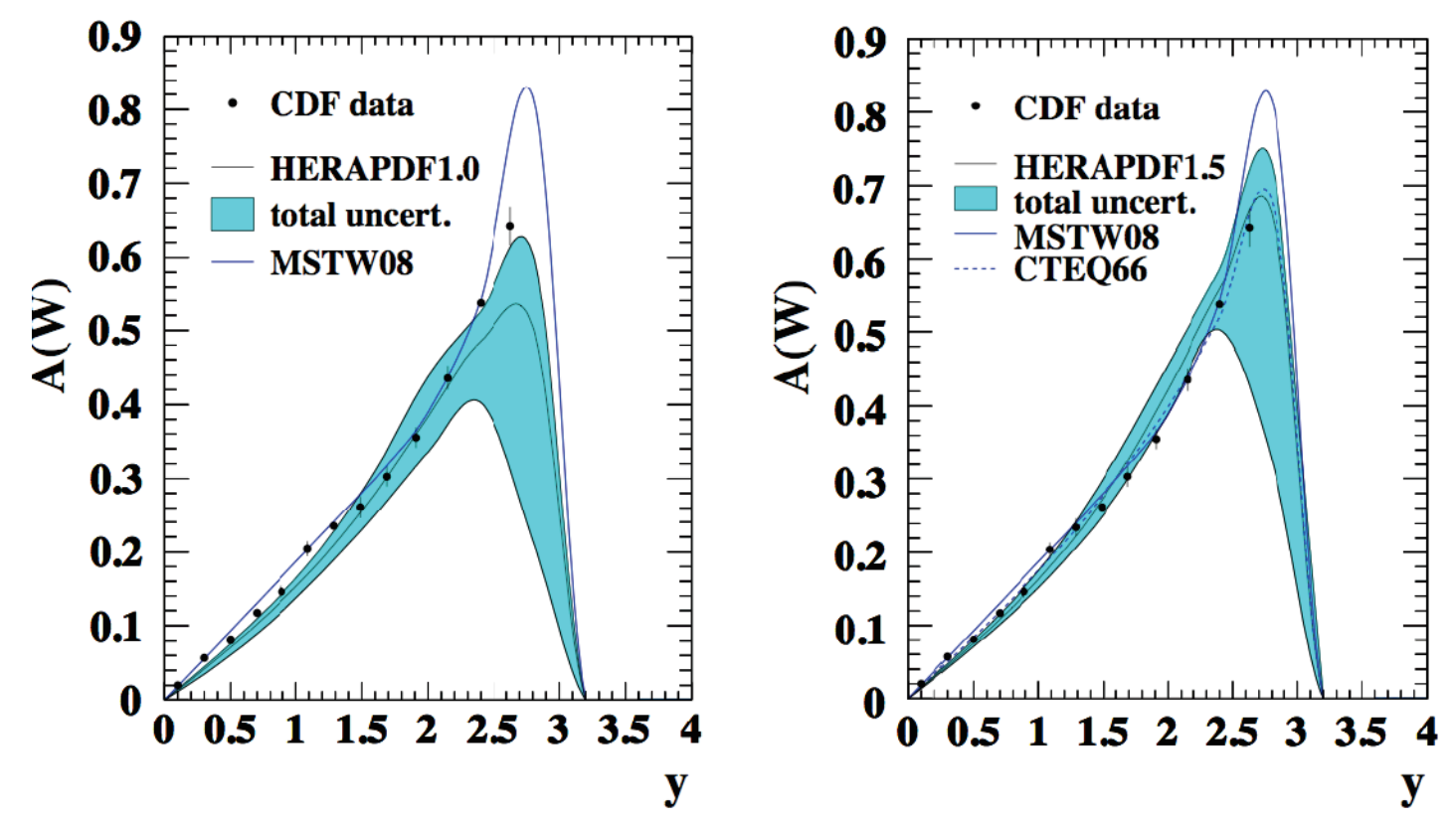

FIGURE 5. Figure shows the CDF data for the $W$ asymmetry compared with HERAPDF1.0 (on the left hand side) and with HERAPDF1.5 (on the right hand side) which results in a better agreement even if this data is not included in the HERA fits as opposed to predictions from CTEQ and MSTW. The HERAPDF predictions include the total unertainties. 


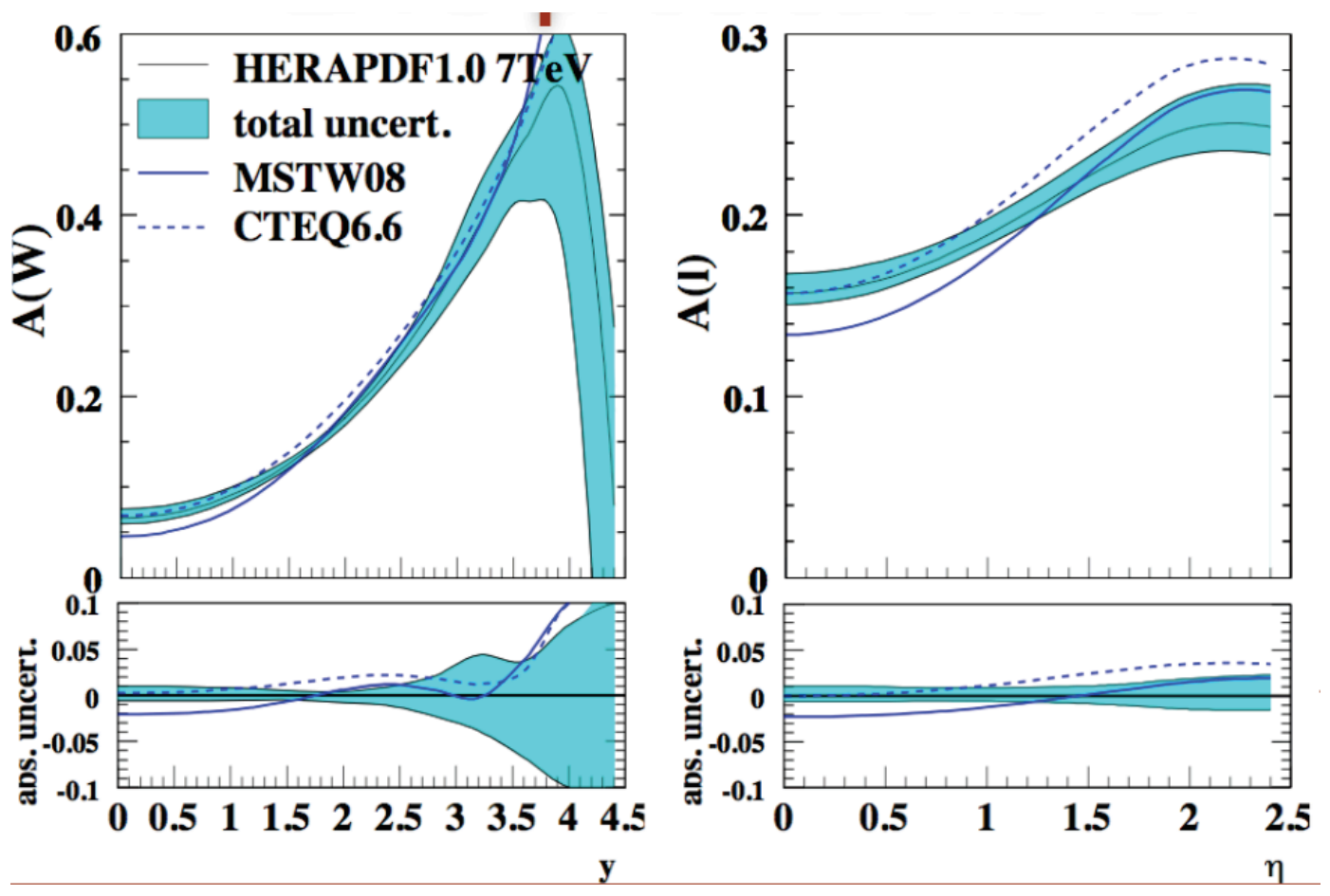

FIGURE 6. Figure shows the LHC predictions for the $W$ and lepton asymmetry, respectively based solely on HERA I data, represented by HERAPDF1.0.
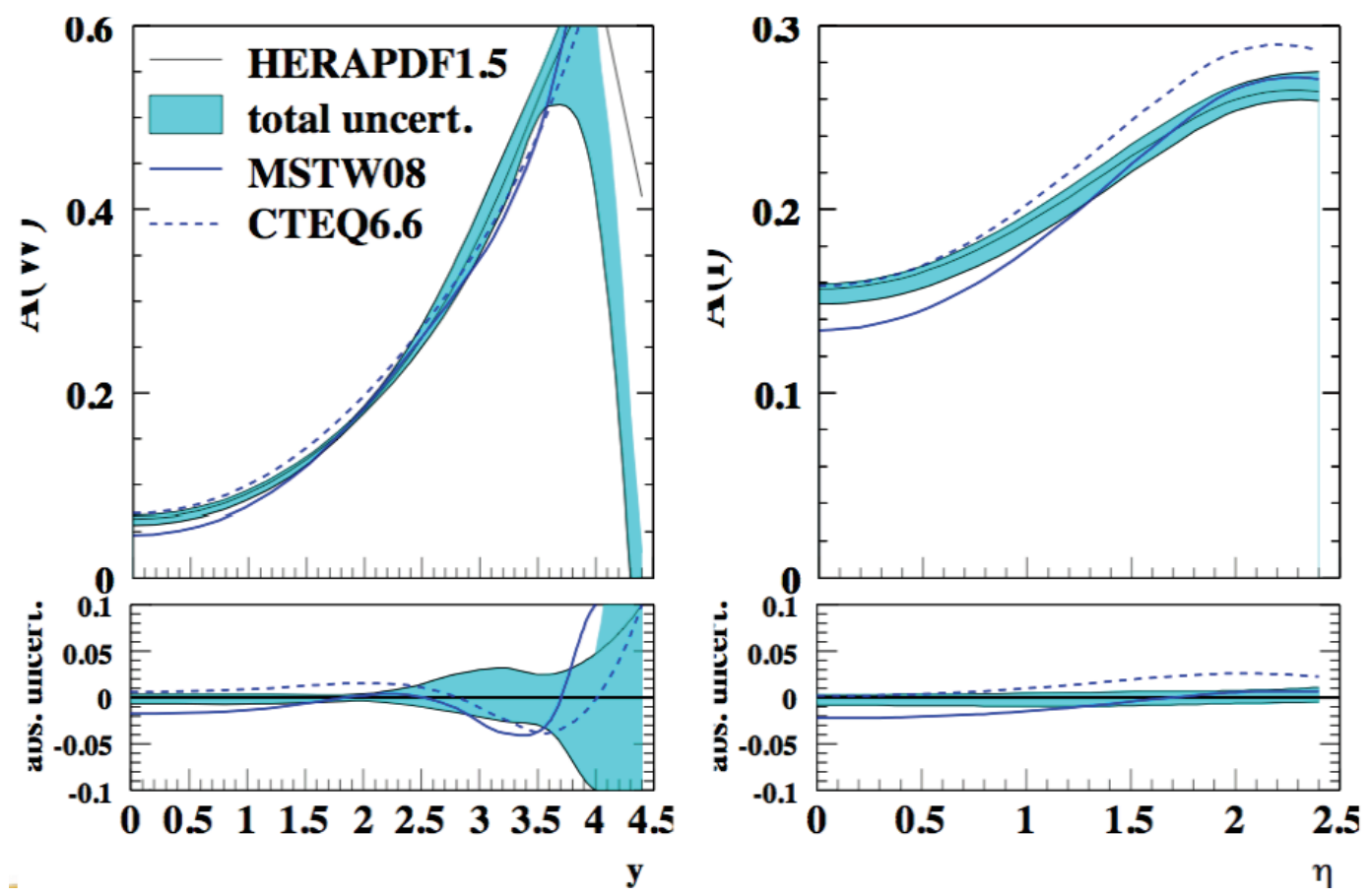

FIGURE 7. Figure shows the impact of the inclusion of HERA II data in HERA PDf fits on the LHC predictions for the Wand lepton asymmetry, respectively. Hence, the uncertainties are reduced forthe predictions using HERAPDF1.5 compared to HERAPDF1.0 shown in Figure 6. 